\title{
Structural and Functional Analysis of Yeast Shu Complex
}

\section{Sam Chu and Hong Ling}

Dept. of Biochemistry, Schulich School of Medicine \& Dentistry, University of Western Ontario, London, ON, Canada.

\begin{abstract}
The error-free DNA lesion bypass pathway is a DNA damage tolerance response that switches replication templates with homologous recombination (HR) to fix DNA damage and ensure genomic authenticity. The spatial and temporal arrangement of proteins in the error-free lesion bypass pathway is largely undefined. The budding yeast Shu complex has been discovered to be crucial for DNA binding and other activities in the HR apparatus. The Shu complex structure has been determined and some key proteins of the error-free lesion bypass pathway have been identified. The molecular basis in which these proteins are organized remains unclear. We are working to characterize the structure and function of the Shu complex interacting with substrates through X-ray crystallography and related functional assays. The binding of specific DNA substrates would cause a conformational change to the Shu complex, which affects its ability to hydrolyze ATP. Structural data will help provide insights into the mechanism of the Shu complex and how it promotes the error-free bypass pathway at the molecular level. DNA substrates with different lengths and end natures were assayed via gel mobility shift and fluorescence polarization/anisotropy to identify substrates that interact best with the Shu complex. Preliminary data generated from malachite green ATPase assays suggest the Shu complex possess ATPase activity that is DNA-binding dependent. Thus far, crystals of the Csm2-Psy3 dimer in complex with DNA diffracted to a resolution of $2.8 \AA$ in the space group of $\mathrm{P}_{3} 12$, which was different from the apo dimer's $\mathrm{C} 2$ space group. It is unclear if the DNA binding influenced the protein's structure without additional evidence from a structure with improved resolution. Additionally, crystals generated from Shu complex incubated with ATP and AMPPNP diffracted in the same $\mathrm{P}_{3} 2_{2} 2$ space group as the published Shu complex structure.

Optimization of the crystallization condition for higher quality data is necessary to confirm the presence/absence of the substrates. In summary, structure built from the DNA-protein complex data could not confirm the DNA substrate relative to the Csm2-Psy3 dimer. Despite the ability to generate crystals, the substrate may not be the most suitable for the complex to consistently occupy a space in the structure. Optimization for better diffraction quality crystals of the Shu complex with ATP/AMP-PNP is underway. Conclusions drawn from this research will lay the foundation for understanding the mechanism of the error-free DNA damage tolerance to aid in combating various diseases like cancers and genetic defects.
\end{abstract}

